\title{
High Performance Networks
}

\author{
José Flich $^{1}$, Alfonso Urso ${ }^{1}$, Ulrich Bruening ${ }^{2}$, and Giuseppe Di Fatta ${ }^{2}$ \\ 1 Topic Chairs \\ 2 Members
}

Interconnection networks are key elements in current scalable compute and storage systems, such as parallel computers, networks of workstations, clusters, and even on-chip interconnects. In all these systems, common aspects of communication are of high interest, including advances in the design, implementation, and evaluation of interconnection networks, network interfaces, topologies, routing algorithms, communication protocols, etc.

This year, five papers discussing some those issues were submitted to this topic. Each paper was reviewed by four reviewers and, finally, we were able to select three regular papers. Although the low submission this year, the three selected papers exhibited the standard quality of past years in the track. The accepted papers discuss interesting issues like the Head-Of-Line removal in fattree topologies, the introduction of new topologies for on-chip networks, and the optimization of algorithms in specific topologies.

In detail, the paper titled An Efficient Strategy for Reducing Head-Of-Line Blocking in Fat-Trees and authored by J. Escudero-Sahuquillo, P. J. Garcia, F. J. Quiles, and J.Duato, discusses and proposes a new queue management strategy for fat-trees, where the number of queues is minimized while guaranteeing good performance levels. In the paper titled A First Approach to King Topologies for On-Chip Networks, authored by E. Stafford, J.L. Bosque, C. Martinez, F. Vallejo, R. Beivide, and C. Camarero, the use of new topologies is tackled for on-chip networks. Finally, paper titledOptimizing Matrix Transpose on Torus Interconnects, and authored by V. T. Chakaravarthy, N. Jain, and Y. Sabharwal, focusing on optimizing matrix transpose on torus interconnects, proposes application-level routing techniques that improve the load balancing, resulting in better performance.

We would like to take the opportunity of thanking the authors who submitted their contribution, as well as the Euro-Par Organizing Committee, and the referees with their highly useful comments, whose efforts have made this conference and this topic possible. 